\title{
Stability Analysis for Mutually Delay-Coupled Semiconductor Lasers System
}

\author{
Rina Su, Chunrui Zhang, and Zuolin Shen \\ Department of Mathematics, Northeast Forestry University, Harbin 150040, China \\ Correspondence should be addressed to Chunrui Zhang; math@nefu.edu.cn \\ Received 19 December 2012; Revised 4 February 2013; Accepted 5 February 2013 \\ Academic Editor: Peixuan Weng
}

Copyright (C) 2013 Rina Su et al. This is an open access article distributed under the Creative Commons Attribution License, which permits unrestricted use, distribution, and reproduction in any medium, provided the original work is properly cited.

In this paper, the problem of the stability for mutually delay-coupled semiconductor lasers system is investigated. By analyzing the associated characteristic equation, linear stability is investigated and Hopf bifurcations are demonstrated. The new phenomena such as stability switch is found. The $Z_{2}$ equivariant property and the existence of multiple periodic solutions is also discussed. Numerical simulations are presented to illustrate the results in the paper.

\section{Introduction}

For many years, coupled nonlinear oscillators have been a source of growing interest in different research fields, ranging from physics, chemistry, and engineering to biology, social sciences and so on [1-5].

Recently, there has been an increasing activity and interest on the study of delay-coupled semiconductor lasers systems, because of their practical importance. The spatial separation of the lasers always results in a time delay in the coupling due to finite signal propagation times [6]. Time delay is ubiquitous in most physical and biological systems like optical bistable devices, electromechanical systems, predatorprey models, and physiological systems. They can arise from finite propagation speeds of signals or from finite processing times in synapses and so on. In many situations, the time delay in the coupling has been neglected. However, for semiconductor lasers this is not always justified due to their large bandwidth and fast time scales of their dynamics.

The objective of [6] is the genetic case of two identical, mutually delay-coupled semiconductor lasers that receive each others light. The authors model the coupled lasers system with rate equations for the normalized complex slowly varying envelope of the optical fields $E_{1,2}$ and the normalized inversions $N_{1,2}$ as follows

$$
\begin{aligned}
\frac{d E_{1}}{d t} & =(1+i \alpha) N_{1} E_{1}+\kappa e^{-i \Omega_{1} \tau_{n}} E_{2}\left(t-\tau_{n}\right), \\
\frac{d E_{2}}{d t} & =(1+i \alpha) N_{2} E_{2}+\kappa e^{-i \Omega_{1} \tau_{n}} E_{1}\left(t-\tau_{n}\right)+i \delta E_{2}, \\
T \frac{d N_{1}}{d t} & =P-N_{1}-\left(1+2 N_{1}\right)\left|E_{1}\right|^{2}, \\
T \frac{d N_{2}}{d t} & =P-N_{2}-\left(1+2 N_{2}\right)\left|E_{2}\right|^{2},
\end{aligned}
$$

where $\kappa>0$ is the coupled strength, $\tau_{n}>0$ is the delay time, $\Omega_{1}$ is the optical frequency of laser 1 operated solitary at threshold, $\Omega_{2}$ is the optical angular frequency of the second laser operated solitary at threshold, and $\delta=\Omega_{1}-\Omega_{2}$. The remaining parameters are the line width enhancement factor $\alpha>0$, the normalized carrier lifetime $T(T>0)$, and the pump parameter $P(P>0)$. The authors obtained experimentally bifurcations and multistabilities between the two lasers. 
In this paper we consider the lasers system (1) with $\Omega_{1}=$ $\Omega_{2}=\Omega>0$, then we have

$$
\begin{aligned}
\frac{d E_{1}}{d t} & =(1+i \alpha) N_{1} E_{1}+\kappa e^{-i \Omega \tau} E_{2}(t-\tau), \\
\frac{d E_{2}}{d t} & =(1+i \alpha) N_{2} E_{2}+\kappa e^{-i \Omega \tau} E_{1}(t-\tau), \\
T \frac{d N_{1}}{d t} & =P-N_{1}-\left(1+2 N_{1}\right)\left|E_{1}\right|^{2}, \\
T \frac{d N_{2}}{d t} & =P-N_{2}-\left(1+2 N_{2}\right)\left|E_{2}\right|^{2} .
\end{aligned}
$$

Consider the complex coupled semiconductor lasers model (2) with a single delay $\tau$. Let $E_{1}=x_{1}-i y_{1}, E_{2}=x_{2}-i y_{2}$, $N_{1}=u_{1}+P-i v_{1}$, and $N_{2}=u_{2}+P-i v_{2}$ be a solution of (2). Then (2) can be rewritten as

$$
\begin{aligned}
& \dot{x}_{1}=\left(u_{1}-\alpha v_{1}+P\right) x_{1}-\left(\nu_{1}+\alpha u_{1}+\alpha P\right) y_{1} \\
& +\kappa \cos (\Omega \tau) x_{2}(t-\tau)+\kappa \sin (\Omega \tau) y_{2}(t-\tau), \\
& \dot{y}_{1}=\left(v_{1}+\alpha u_{1}+\alpha P\right) x_{1}-\left(u_{1}-\alpha v_{1}+P\right) y_{1} \\
& -\kappa \sin (\Omega \tau) x_{2}(t-\tau)+\kappa \sin (\Omega \tau) y_{2}(t-\tau), \\
& \dot{x}_{2}=\left(u_{2}-\alpha v_{2}+P\right) x_{2}-\left(\nu_{2}+\alpha u_{2}+\alpha P\right) y_{2} \\
& +\kappa \cos (\Omega \tau) x_{1}(t-\tau)+\kappa \sin (\Omega \tau) y_{1}(t-\tau), \\
& \dot{y}_{2}=\left(v_{2}+\alpha u_{2}+\alpha P\right) x_{2}+\left(u_{2}-\alpha v_{2}+P\right) y_{2} \\
& -\kappa \sin (\Omega \tau) x_{1}(t-\tau)+\kappa \cos (\Omega \tau) y_{1}(t-\tau), \\
& \dot{u}_{1}=-\frac{1}{T}\left[u_{1}+x_{1}^{2}-y_{1}^{2}+2 u_{1} x_{1}^{2}+2 P x_{1}^{2}\right. \\
& \left.-2 u_{1} y_{1}^{2}-2 P y_{1}^{2}-4 v_{1} x_{1} y_{1}\right] \\
& \dot{v}_{1}=-\frac{1}{T}\left[v_{1}+2 x_{1} y_{1}+4 u_{1} x_{1} y_{1}\right. \\
& \left.+4 P x_{1} y_{1}+2 v_{1} x_{1}^{2}-2 v_{1} y_{1}^{2}\right] \\
& \dot{u}_{2}=-\frac{1}{T}\left[u_{2}+x_{2}^{2}-y_{2}^{2}+2 u_{2} x_{2}^{2}+2 P x_{2}^{2}\right. \\
& \left.-2 u_{2} y_{2}^{2}-2 P y_{2}^{2}-4 v_{2} x_{2} y_{2}\right] \\
& \dot{v}_{2}=-\frac{1}{T}\left[v_{2}+2 x_{2} y_{2}+4 u_{2} x_{2} y_{2}\right. \\
& \left.+4 P x_{2} y_{2}+2 v_{2} x_{2}^{2}-2 v_{2} y_{2}^{2}\right] \text {. }
\end{aligned}
$$

The purpose of this paper is to study the properties of stability and bifurcation in model (3). Because of the optical frequencies are the same, which leads to a detuning between the lasers, for achieving high out-put power, the effect of the time delay between the semiconductor lasers has been more and more importance. In our study, we need to found that when the time delay reach a certain value, arbitrarily small perturbation will make the dynamic performance of the system (3) have changed. That is to say, if the time delay is in a certain range, the out-put power is stable or unstable.

We also find some new phenomena such as stability switch for (3) which is not mentioned in [6]. Couple can lead synchronization, phase trapping, phase locking, amplitude death, chaos, bifurcation of oscillators and so on [7-9]. Since two identical oscillators are coupled symmetrically, then the most typical patterns of behavior are perfect synchrony or perfect antisynchrony (in which the oscillators are half a period out of phase with each other). In Section 3, we give the $Z_{2}$-equivariant property of (3) and the existence of multiple periodic solutions (synchronous respectively, anti-phased).

The paper is organized as follows. In Section 2, we analyze the distribution of the characteristic equation associated with multicoupled, and obtain the existence of the local Hopf bifurcation and stability of the bifurcating periodic solutions. Base on the symmetric bifurcation theorem of Golubitsky [10], we also discussed the $Z_{2}$ equivariant property and the existence of multiple periodic solutions in Section 3. To verify the theoretic analysis, numerical simulations are given in Section 4.

\section{Stability Analysis}

It is clear that the origin $(0,0,0,0,0,0,0,0)$ is a stationary point of (3). The linearization of (3) at the origin $(0,0,0,0$, $0,0,0,0)$ is

$$
\begin{aligned}
\dot{x}_{1}= & P x_{1}-\alpha P y_{1}+\kappa \cos (\Omega \tau) \\
& \quad \times x_{2}(t-\tau)+\kappa \sin (\Omega \tau) y_{2}(t-\tau), \\
\dot{y}_{1}=\alpha & P x_{1}-P y_{1}-\kappa \sin (\Omega \tau) \\
& \quad \times x_{2}(t-\tau)+\kappa \sin (\Omega \tau) y_{2}(t-\tau), \\
\dot{x}_{2}=P x_{2}-\alpha P y_{2}+\kappa \cos (\Omega \tau) & \quad \times x_{1}(t-\tau)+\kappa \sin (\Omega \tau) y_{1}(t-\tau), \\
\dot{y}_{2}=\alpha & P x_{2}+P y_{2}-\kappa \sin (\Omega \tau) \\
& \times x_{1}(t-\tau)+\kappa \cos (\Omega \tau) y_{1}(t-\tau), \\
\dot{u}_{1}=- & \frac{1}{T} u_{1}, \\
\dot{v}_{1}=- & \frac{1}{T} \nu_{1}, \\
\dot{u}_{2}=- & \frac{1}{T} u_{2}, \\
\dot{v}_{2}=- & \frac{1}{T} \nu_{2} .
\end{aligned}
$$

Among them, the $\kappa>0$ is a coupling strength, $P>0, \alpha>0$, $\Omega>0$, and $T>0$. The zero solution of system (4) is asymptotically stable if and only if all the roots of the characteristic equation associated with system (4) have negative real parts. The characteristic equation of system (4) is

$$
\operatorname{det} \mu_{1}(\lambda) \cdot \operatorname{det} \mu_{2}(\lambda)=0
$$


where

$$
\begin{aligned}
& \operatorname{det} \mu_{1}(\lambda) \\
& =\left|\begin{array}{cccc}
\lambda-P & \alpha P & -\kappa e^{-\lambda \tau} \cos (\Omega \tau) & -\kappa e^{-\lambda \tau} \sin (\Omega \tau) \\
-\alpha P & \lambda-P & \kappa e^{-\lambda \tau} \sin (\Omega \tau) & -\kappa e^{-\lambda \tau} \cos (\Omega \tau) \\
-\kappa e^{-\lambda \tau} \cos (\Omega \tau) & -\kappa e^{-\lambda \tau} \sin (\Omega \tau) & \lambda-P & \alpha P \\
\kappa e^{-\lambda \tau} \sin (\Omega \tau) & -\kappa e^{-\lambda \tau} \cos (\Omega \tau) & -\alpha P & \lambda-P
\end{array}\right|, \\
& \operatorname{det} \mu_{2}(\lambda)=\left|\begin{array}{cccc}
\lambda+\frac{1}{T} & & \\
& \lambda+\frac{1}{T} & \\
& & \lambda+\frac{1}{T} & \\
& & & \lambda+\frac{1}{T}
\end{array}\right|
\end{aligned}
$$

by a direct calculation, and one can obtain that

$$
\begin{aligned}
& \operatorname{det} \mu_{1}(\lambda) \\
& =\left[\left(\lambda-P-\kappa e^{-\lambda \tau} \cos (\Omega \tau)\right)^{2}+\left(\alpha P-\kappa e^{-\lambda \tau} \sin (\Omega \tau)\right)^{2}\right] \\
& \times\left[\left(\lambda-P+\kappa e^{-\lambda \tau} \cos (\Omega \tau)\right)^{2}+\left(\alpha P+\kappa e^{-\lambda \tau} \sin (\Omega \tau)\right)^{2}\right] \\
& \operatorname{det} \mu_{2}(\lambda)=\left(\lambda+\frac{1}{T}\right)^{4} .
\end{aligned}
$$

It is well known from the theory of functional differential equations that characteristic equation (5) determines local stability of the trivial solution of (3). Thus, we need to investigate the distribution of roots of the characteristic equation (5). To achieve this aim, it is convenient to consider the following.

Clearly, (7) can be written as

$$
\operatorname{det} \mu_{1}(\lambda)=\Delta^{-}(\lambda) \cdot \Delta^{+}(\lambda)
$$

where

$$
\Delta^{-}(\lambda)
$$

$$
=\left(\lambda-P-\kappa e^{-\lambda \tau} \cos (\Omega \tau)\right)^{2}+\left(\alpha P-\kappa e^{-\lambda \tau} \sin (\Omega \tau)\right)^{2},
$$

$\Delta^{+}(\lambda)$

$$
=\left(\lambda-P+\kappa e^{-\lambda \tau} \cos (\Omega \tau)\right)^{2}+\left(\alpha P+\kappa e^{-\lambda \tau} \sin (\Omega \tau)\right)^{2} .
$$

Since $\operatorname{det} \mu_{1}(\lambda) \cdot \operatorname{det} \mu_{2}(\lambda)=0$, then we have $\operatorname{det} \mu_{1}(\lambda)=0$ or $\operatorname{det} \mu_{2}(\lambda)=0$. If $\operatorname{det} \mu_{2}(\lambda)=0$, we have $\lambda=-1 / T$, and then all roots of the characteristic equation (5) have negative real parts. In this case, they have no influence on the system (3); then system (3) is stable. We will omit it.

So, we just consider

$$
\operatorname{det} \mu_{1}(\lambda)=0
$$

It is easy to verify that $\lambda$ is a root of (11) if and only if it is a root of one of the following equations:

$$
\begin{aligned}
& \lambda-P-\kappa e^{-\lambda \tau} \cos (\Omega \tau)=i\left(\alpha P-\kappa e^{-\lambda \tau} \sin (\Omega \tau)\right), \\
& \lambda-P-\kappa e^{-\lambda \tau} \cos (\Omega \tau)=-i\left(\alpha P-\kappa e^{-\lambda \tau} \sin (\Omega \tau)\right), \\
& \lambda-P+\kappa e^{-\lambda \tau} \cos (\Omega \tau)=i\left(\alpha P+\kappa e^{-\lambda \tau} \sin (\Omega \tau)\right), \\
& \lambda-P+\kappa e^{-\lambda \tau} \cos (\Omega \tau)=-i\left(\alpha P+\kappa e^{-\lambda \tau} \sin (\Omega \tau)\right),
\end{aligned}
$$

and it is not difficult to verify that $\lambda=q+i \beta$ is a root of (12) if and only if $\lambda=q-i \beta$ is a root of (13), and $\lambda=q+i \beta$ is a root of (14) if and only if $\lambda=q-i \beta$ is a root of (15). Therefore, it is sufficient to investigate only (12) and (14). We next want to find the conditions which determine that all roots of (12)-(15) satisfy $\operatorname{Re}(\lambda)<0$.

We first consider (12), clearly $\lambda=0$ is not a root of (3). Let $\lambda=i \beta,(\beta \neq 0)$ be a root of $(12)$, then $\beta$ satisfies

$$
\begin{aligned}
& \kappa \cos (\beta \tau+\Omega \tau)=-P, \\
& \kappa \sin (\beta \tau+\Omega \tau)=\alpha P-\beta,
\end{aligned}
$$

and it follows that

$$
(\alpha P-\beta)^{2}=\kappa^{2}-P^{2}
$$

Clearly, (17) has no real root if $0<\kappa<P$ and has two real roots $\beta_{ \pm}$if $\kappa>P$, where

$$
\beta_{ \pm}=\alpha P \pm \sqrt{\kappa^{2}-P^{2}} .
$$

Consequently by (16), we can state the following results.

Lemma 1. (1) If $0<\kappa<P$, then (12) has no purely imaginary root.

(2) If $\kappa>P$, then we have the following.

(i) For $P<\kappa<\sqrt{\alpha^{2} P^{2}+P^{2}}$, define

$$
\begin{aligned}
& \bar{\tau}_{j}^{+}=\frac{\pi+\arccos (P / \kappa)+2 j \pi}{\beta_{+}+\Omega}, \\
& \bar{\tau}_{j}^{-}=\frac{\pi-\arccos (P / \kappa)+2 j \pi}{\beta_{-}+\Omega},
\end{aligned}
$$

where $j=0,1,2, \ldots$, then $i \beta_{ \pm}$are purely imaginary roots of (12), with $\tau=\bar{\tau}_{j}^{ \pm}$, respectively.

(ii) If $\kappa>\sqrt{\alpha^{2} P^{2}+P^{2}} \cap\left(\beta_{-}+\Omega\right)<0$, then (12) has purely imaginary roots $i \beta_{ \pm}$when $\tau=\bar{\tau}_{j}^{ \pm}$, respectively, where $\bar{\tau}_{j}^{+}$ is defined by (19) and

$$
\bar{\tau}_{j}^{-}=\frac{\pi+\arccos (P / \kappa)+2 j \pi}{-\left(\beta_{-}+\Omega\right)} .
$$

(iii) If $\kappa=\sqrt{\alpha^{2} P^{2}+P^{2}}$, then i $\beta_{+}$is a purely imaginary root of (12) with $\tau=\bar{\tau}_{j}^{+}$, where $\beta_{+}=2 \alpha p$ and $\bar{\tau}_{j}^{+}$is defined by (19). 
(3) If $\kappa=P$, then $i \beta_{+}$is a purely imaginary root of (12) with $\tau=\bar{\tau}_{j}^{+}$, where $\beta_{+}=\alpha p$ and $\bar{\tau}_{j}^{+}=(2(j+1) \pi) /(\alpha P+\Omega),(j=$ $0,1,2, \ldots)$.

Lemma 2. If $\kappa>P$, then $\operatorname{Re}(d \lambda / d \tau)_{\tau=\bar{\tau}_{j}^{+}}>0$, and

$$
\operatorname{Re}\left(\frac{d \lambda}{d \tau}\right)_{\tau=\bar{\tau}_{j}^{-}} \begin{cases}<0, & \text { for } P<\kappa<\sqrt{\alpha^{2} P^{2}+P^{2}}, \\ >0, & \text { for } \kappa>\sqrt{\alpha^{2} P^{2}+P^{2}} \cap\left(\beta_{-}+\Omega\right)<0,\end{cases}
$$

where $j=0,1,2, \ldots$..

Proof. Substituting $\lambda(\tau)$ into (12) and taking the derivative with the respect to $\tau$, we can easily obtain

$$
\left[\frac{d \lambda}{d \tau}\right]^{-1}=\frac{1}{\kappa e^{-\lambda \tau}(1+i \Omega)(i \sin (\Omega \tau)-\cos (\Omega \tau))}-\frac{\tau}{\lambda+i \Omega} .
$$

Note that $\beta_{+}>0$, and $\beta_{-}>0$ when $P<\kappa<\sqrt{\alpha^{2} P^{2}+P^{2}}$, while $\beta_{-}<0 \cap\left(\beta_{-}+\Omega\right)<0$ when $\kappa>\sqrt{\alpha^{2} P^{2}+P^{2}}$. Thus by (16) and (23), we deduce that

$$
\begin{gathered}
\operatorname{Re}\left[\frac{d \lambda}{d \tau}\right]_{\tau=\bar{\tau}_{j}^{+}}^{-1}=\frac{\beta_{+}-\alpha P}{\kappa^{2}\left(\beta_{+}+\Omega\right)}>0, \\
\operatorname{Re}\left(\frac{d \lambda}{d \tau}\right)_{\tau=\bar{\tau}_{j}}^{-1}=\frac{\beta_{-}-\alpha P}{\kappa^{2}\left(\beta_{-}+\Omega\right)} \\
= \begin{cases}<0, & P<\kappa<\sqrt{\alpha^{2} P^{2}+P^{2}}, \\
>0, & \kappa>\sqrt{\alpha^{2} P^{2}+P^{2}} \cap\left(\beta_{-}+\Omega\right)<0 .\end{cases}
\end{gathered}
$$

Since the sign of $\operatorname{Re}(d \lambda / d \tau)$ is same as that of $\operatorname{Re}(d \lambda / d \tau)^{-1}$, this lemma follows immediately.

Combining (12) and (13) as follows:

$$
\lambda-P-\kappa e^{-\lambda \tau} \cos (\Omega \tau)= \pm i\left(\alpha P-\kappa e^{-\lambda \tau} \sin (\Omega \tau)\right) .
$$

Then Lemmas 1 and 2 allow us to state and prove the following results.

Lemma 3. Assume that $\beta_{ \pm}$are defined by (18) and $\bar{\tau}_{j}^{ \pm}$are shown as in Lemma 1.

(1) If $0<\kappa<P$, then (25) has two roots with positive real parts for all $\tau \geq 0$.

(2) If $P<\kappa<\sqrt{\alpha^{2} P^{2}+P^{2}}$ and $\arccos (P / \kappa)>$ $\left(\sqrt{\kappa^{2}-P^{2}} /(\alpha P+\Omega)\right) \pi$, then there exists an integer $m$ such that $0<\bar{\tau}_{0}^{-}<\bar{\tau}_{0}^{+}<\cdots<\bar{\tau}_{m-1}^{-}<\bar{\tau}_{m-1}^{+}<$ $\bar{\tau}_{m}^{-}<\bar{\tau}_{m}^{+}<\bar{\tau}_{m+1}^{+}<\bar{\tau}_{m+1}^{-}$. In this case, all roots of (25) have negative real parts when $\tau \in \bigcup_{j=0}^{m}\left(\bar{\tau}_{j}^{-} \quad \bar{\tau}_{j}^{+}\right),(25)$ has a pair of roots with positive real parts when $\tau \in$ $\bigcup_{j=0}^{m}\left(\bar{\tau}_{(j-1)}^{+} \bar{\tau}_{j}^{-}\right)$where $\bar{\tau}_{(-1)}^{+}=0$, and (25) has at least a pair of roots with positive real parts when $\tau>\bar{\tau}_{m}^{+}$. In addition, when $\tau=\bar{\tau}_{j}^{+}$and $\tau=\bar{\tau}_{j}^{-},(j=0,1,2, \ldots)$, all roots of (25) have negative real parts except the purely imaginary roots $\pm i \beta_{+}$and $\pm i \beta_{-}$, respectively.
(3) If $P<\kappa<\sqrt{\alpha^{2} P^{2}+P^{2}}$ and $\arccos (P / \kappa)<$ $\left(\sqrt{\kappa^{2}-P^{2}} /(\alpha P+\Omega)\right) \pi$, then there exists an integer $n$ such that $0<\bar{\tau}_{0}^{+}<\bar{\tau}_{0}^{-}<\cdots<\bar{\tau}_{n-1}^{+}<\bar{\tau}_{n-1}<$ $\bar{\tau}_{n}^{+}<\bar{\tau}_{n+1}^{+}<\bar{\tau}_{n}^{-}$. In this case, all roots of (25) have negative real parts when $\tau \in\left[\begin{array}{ll}0 & \bar{\tau}_{0}^{+}\end{array}\right) \cup \bigcup_{j=0}^{n}\left(\begin{array}{ll}\bar{\tau}_{j-1}^{-} & \bar{\tau}_{j}^{+}\end{array}\right)$, (25) has a pair of roots with positive real parts when $\tau \in \bigcup_{j=0}^{n-1}\left(\bar{\tau}_{j}^{+} \quad \bar{\tau}_{j}\right)$ and (25) has at least a pair of roots with positive real parts when $\tau>\bar{\tau}_{n}^{+}$. In addition, when $\tau=\bar{\tau}_{j}^{+},(j=0,1,2, \ldots, n)$ and $\tau=\bar{\tau}_{j}^{-},(j=$ $0,1,2, \ldots, n-1)$, all roots of $(25)$ have negative real parts except the purely imaginary roots $\pm i \beta_{+}$and $\pm i \beta_{-}$, respectively.

(4) If $\kappa>\sqrt{\alpha^{2} P^{2}+P^{2}} \bigcap\left(\beta_{-}+\Omega\right)<0$, then (25) has at least two part roots with positive real parts for all $\tau \geq 0$.

Proof. (1) When $\tau=0$, (25) becomes $\lambda=P+\kappa \pm i \alpha P$, it is easy to see that (25) with $\tau=0$ has only two roots with positive real parts.

This shows that (25) has no purely imaginary root. Meanwhile, $\tau=0$ is not a root of (25). Thus there is no root appearing on the imaginary axis. Hence (25) has two roots with positive real parts for all $\tau \geq 0$. The proof is complete.

In what follows, we only prove the conclusions in case (2). In case (3), the proof is similar. We just omit it.

(2) When $P<\kappa<\sqrt{\alpha^{2} P^{2}+P^{2}}$ and $\arccos (P / \kappa)>$ $\left(\sqrt{\kappa^{2}-P^{2}} /(\alpha P+\Omega)\right) \pi$, it is not difficult to verify that, $\bar{\tau}_{0}^{+}>\overline{\tau_{0}^{-}}$ and $\left|\bar{\tau}_{(j+1)}^{+}-\bar{\tau}_{j}^{+}\right|<\left|\bar{\tau}_{(j+1)}^{-}-\bar{\tau}_{j}\right|, \tau_{0}=\bar{\tau}_{0}$ is the first value of $\tau \geq 0$ such that (25) has imaginary root. From Lemma 2, we know that

$$
\begin{aligned}
& \operatorname{Re}\left(\frac{d \lambda}{d \tau}\right)_{\tau=\bar{\tau}_{j}^{+}}>0, \\
& \operatorname{Re}\left(\frac{d \lambda}{d \tau}\right)_{\tau=\bar{\tau}_{j}^{-}}<0,
\end{aligned}
$$

we have that all the roots of (25) have negative real parts when $\tau \in \bigcup_{j=0}^{m}\left(\bar{\tau}_{j}^{-} \bar{\tau}_{j}^{+}\right)$. Therefore, together with Lemmas 1 and 2, means that the lemma is true.

In a similar way, for the equation

$$
\lambda-P+\kappa e^{-\lambda \tau} \cos (\Omega \tau)= \pm i\left(\alpha P+\kappa e^{-\lambda \tau} \sin (\Omega \tau)\right),
$$

which is the combination of (14) and (15), we can show the lemma below.

Lemma 4. (1) If $0<\kappa<P$, then (27) has no purely imaginary root for all $\tau \geq 0$.

(2) Assume that $\kappa>P$, then we have the following.

(i) If $P<\kappa<\sqrt{\alpha^{2} P^{2}+P^{2}}$, then (27) has a pair of purely imaginary roots $\pm i \beta_{ \pm}$when $\tau=\tau_{j}^{ \pm},(j=0,1,2, \ldots)$, respectively, and

$$
\begin{gathered}
\tilde{\tau}_{j}^{+}=\frac{\arccos (P / \kappa)+2 j \pi}{\beta_{+}+\Omega}, \\
\tilde{\tau}_{j}^{-}=\frac{2 \pi-\arccos (P / \kappa)+2 j \pi}{\beta_{-}+\Omega} .
\end{gathered}
$$


(ii) If $\kappa>\sqrt{\alpha^{2} P^{2}+P^{2}} \bigcap\left(\beta_{-}+\Omega\right)<0$, then (27) has a pair of purely imaginary roots $\pm i \beta_{ \pm}$when $\tau=\tau_{j}^{ \pm}$, respectively, where $\beta_{ \pm}=\alpha P \pm \sqrt{\kappa^{2}-P^{2}}, \tilde{\tau}_{j}^{+}$is defined by (28) and

$$
\tilde{\tau}_{j}^{-}=\frac{\arccos (P / \kappa)+2 j \pi}{-\left(\beta_{-}+\Omega\right)} .
$$

(iii) If $\kappa=\sqrt{\alpha^{2} P^{2}+P^{2}}$, then (27) has a pair of purely imaginary roots $\pm i \beta_{+}$when $\tau=\tilde{\tau}_{j}^{+},(j=0,1,2, \ldots)$, where $\beta_{+}=2 \alpha p$ and $\tilde{\tau}_{j}^{+}$is defined by (28).

(3) If $\kappa=P$, then (27) has a pair of purely imaginary roots $\pm i \beta_{+}$when $\tau=\tilde{\tau}_{j}^{+}$, where $\beta_{+}=\alpha p$ and $\tilde{\tau}_{j}^{+}=((2 j+1) \pi) /(\alpha P+$ $\Omega),(j=0,1,2, \ldots)$.

By a direct computation, we can obtain that if $\kappa>P$, then

$$
\begin{gathered}
\operatorname{Re}\left(\frac{d \lambda}{d \tau}\right)_{\tau=\tilde{\tau}_{j}^{+}}>0, \\
\operatorname{Re}\left(\frac{d \lambda}{d \tau}\right)_{\tau=\tilde{\tau}_{j}^{-}} \begin{cases}<0, & \text { for } P<\kappa<\sqrt{\alpha^{2} P^{2}+P^{2}} \\
>0, & \text { for } \kappa>\sqrt{\alpha^{2} P^{2}+P^{2}} \cap\left(\beta_{-}+\Omega\right)<0 .\end{cases}
\end{gathered}
$$

In addition, it is easy to see that (27) has a pair of roots with negative real parts for $\kappa>P$ and has only two roots with positive real parts for $0<\kappa<P$, with $\tau=0$. Consequently, by Lemma 4 and (31), we can show the lemma below.

Lemma 5. Assume that $\beta_{ \pm}$are defined by (18) and $\tilde{\tau}_{j}^{ \pm}$are shown as in Lemma 4.

(1) If $0<\kappa<P$, then (27) has two roots with positive real parts for all $\tau \geq 0$.

(2) If $P<\kappa<\sqrt{\alpha^{2} P^{2}+P^{2}}$, then there exists an integer $\ell$ such that $0<\widetilde{\tau}_{0}^{+}<\widetilde{\tau}_{0}^{-}<\cdots<\tilde{\tau}_{\ell-1}^{+}<\widetilde{\tau}_{\ell-1}^{-}<\widetilde{\tau}_{\ell}^{+}<$ $\tilde{\tau}_{\ell+1}^{+}<\tilde{\tau}_{\ell}^{-}$. In this case, all roots of $(27)$ have negative real parts when $\tau \in\left[\begin{array}{ll}0 & \tilde{\tau}_{0}^{+}\end{array}\right) \cup \tau \in \bigcup_{j=1}^{\ell}\left(\begin{array}{ll}\tilde{\tau}_{j-1}^{-} & \tilde{\tau}_{j}^{+}\end{array}\right)$, (27) has a pair of roots with positive real parts when $\tau \in \bigcup_{j=0}^{\ell}\left(\tilde{\tau}_{j}^{+} \tilde{\tau}_{j}^{-}\right)$, and (27) has at least a pair of roots with positive real parts when $\tau>\tilde{\tau}_{\ell}^{+}$. Moreover, all roots of (27) have negative real parts except the purely imaginary roots $\pm i \beta_{+}$for $\tau=\widetilde{\tau}_{j}^{+},(j=0,1,2, \ldots, \ell)$ and $\pm i \beta_{-}$for $\tau=\tilde{\tau}_{j}^{-},(j=0,1,2, \ldots, \ell-1)$.

(3) If $\kappa>\sqrt{\alpha^{2} P^{2}+P^{2}} \bigcap\left(\beta_{-}+\Omega\right)<0$, then all roots of (27) has at least two part roots with positive real parts for all $\tau \geq \widetilde{\tau}_{0}^{+}$, and (27) has a pair of purely imaginary roots $\pm i \beta_{ \pm}$when $\tau=\tilde{\tau}_{j}^{ \pm}$, respectively.

Now, we are in a position to study (13). It is easy to see from Lemmas 3 and 5 that (13) has at least one root with positive real part when $0<\kappa<P$ or $\kappa>\sqrt{\alpha^{2} P^{2}+P^{2}}$. However, when $P<\kappa<\sqrt{\alpha^{2} P^{2}+P^{2}}$, a more interesting phenomenon such as stability switches, the stability of the equilibrium switching from instability to stability and back to instability or from stability to instability and back to stability several times may occur. In this case, for the convenience of statement, by (19) and (28), we can rearrange $\bar{\tau}_{j}^{+}, \widetilde{\tau}_{j}^{+}$as follows:

$$
\tau_{j}^{+}=\frac{\pi+\arccos (P / \kappa)+j \pi}{\beta_{+}+\Omega},
$$

and by (20) and (29), we can rearrange $\bar{\tau}_{j}^{-}, \widetilde{\tau}_{j}^{-}$as follows

$$
\tau_{j}^{-}=\frac{\pi-\arccos (P / \kappa)+j \pi}{\beta_{-}+\Omega},
$$

where $j=0,1,2, \ldots$. By Lemma 2 and (31), we can easily obtain the following results on the distribution of zeros of the characteristic (11).

Lemma 6. Assume that $\beta_{ \pm}$are defined by (18) and $\tau_{j}^{ \pm}$are defined by (32)-(33), respectively.

(1) If $0<\kappa<P$, then (11) has four roots with positive real parts for all $\tau \geq 0$.

(2) If $P<\kappa<\sqrt{\alpha^{2} P^{2}+P^{2}}$ and $\arccos (P / \kappa)<$ $\left(\left(\sqrt{\kappa^{2}-P^{2}}\right) /(\alpha P+\Omega)\right) \pi$, then there exists an integer $h$ such that $0<\tau_{0}^{+}<\tau_{0}^{-}<\cdots<\tau_{h-1}^{+}<\tau_{h-1}^{-}<$ $\tau_{h}^{+}<\tau_{h+1}^{+}<\tau_{h}^{-}$. Moreover, all roots of (11) has negative real parts when $\tau \in\left[\begin{array}{ll}0 & \bar{\tau}_{0}^{+}\end{array}\right) \cup \tau \in \bigcup_{j=1}^{h}\left(\begin{array}{ll}\tau_{(j-1)}^{-} & \tau_{j}^{+}\end{array}\right)$, (11) has a pair of roots with positive real parts when $\tau \in \bigcup_{j=0}^{h-1}\left(\tau_{j}^{+} \tau_{j}^{-}\right)$, and (11) has at least a pair of roots with positive real parts when $\tau>\tau_{h}^{+}$. In addition, when $\tau=\tau_{j}^{+},(j=0,1,2, \ldots, h)$ and $\tau=\tau_{j}^{-},(j=$ $0,1,2, \ldots, h-1)$, all roots of (11) have negative real parts except the purely imaginary roots $\pm i \beta_{+}$and $\pm i \beta_{-}$, respectively.

(3) If $P<\kappa<\sqrt{\alpha^{2} P^{2}+P^{2}}$ and $\arccos (P / \kappa)>$ $\left(\sqrt{\kappa^{2}-P^{2}} /(\alpha P+\Omega)\right) \pi$, then there exists an integer $g$ such that $0<\tau_{0}^{-}<\tau_{0}^{+}<\cdots<\tau_{g-1}^{-}<\tau_{g-1}^{+}<$ $\tau_{g}^{-}<\tau_{g}^{+}<\tau_{g+1}^{+}<\tau_{g+1}^{-}$. Moreover, all roots of (11) has negative real parts for $\tau \in \bigcup_{j=0}^{g}\left(\tau_{(j-1)}^{+} \quad \tau_{j}^{-}\right)$, where $\tau_{-1}^{+}=0$; and (11) has at least a pair of roots with positive real parts for $\tau>\tau_{j}^{+}$. In addition, when $\tau=\tau_{j}^{+}$ and $\tau=\tau_{j}^{-},(j=0,1,2, \ldots, g)$, all roots of (11) have negative real parts except the purely imaginary roots $\pm i \beta_{+}$and $\pm i \beta_{-}$, respectively.

(4) If $\kappa>\sqrt{\alpha^{2} P^{2}+P^{2}} \bigcap\left(\beta_{-}+\Omega\right)<0$, then (11) has at least two roots with positive real parts for all $\tau \geq 0$. Moreover, when $\tau=\bar{\tau}_{j}^{ \pm}$(11) has a pair of purely imaginary roots $\pm i \beta_{+}$, respectively, and when $\tau=\widetilde{\tau}_{j}^{ \pm}$(11) has a pair of purely imaginary roots $\pm i \beta_{ \pm}$, respectively. Here $\bar{\tau}_{j}^{ \pm}$and $\tilde{\tau}_{j}^{ \pm}$are shown as in Lemmas 1 and 4 , respectively.

Lemmas 2 and 6, together with (31), allow us to state the following results on the stability of the zero equilibrium of mutually delay-coupled system (3) and Hopf bifurcations. 


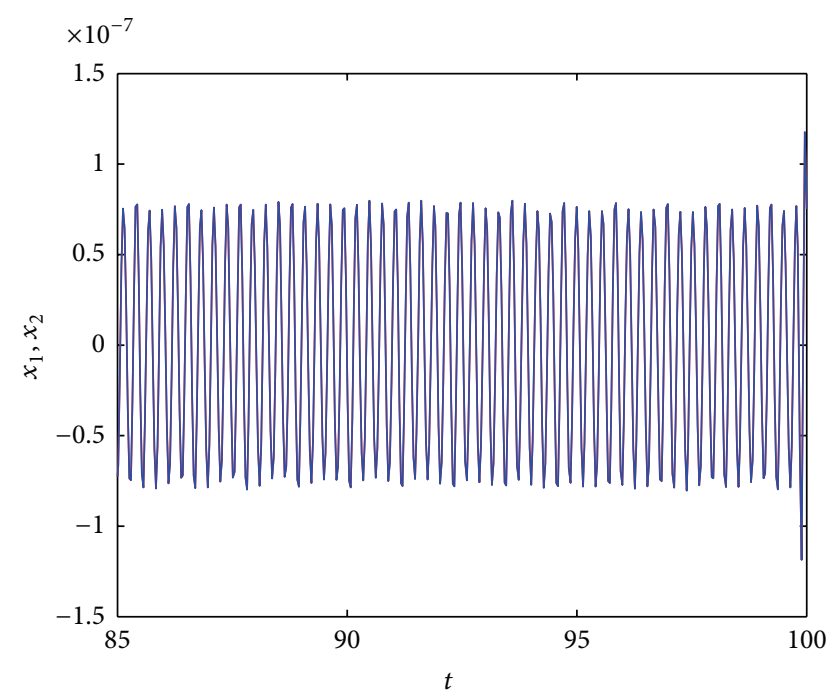

$\begin{aligned} & x_{1} \\ &- x_{2}\end{aligned}$

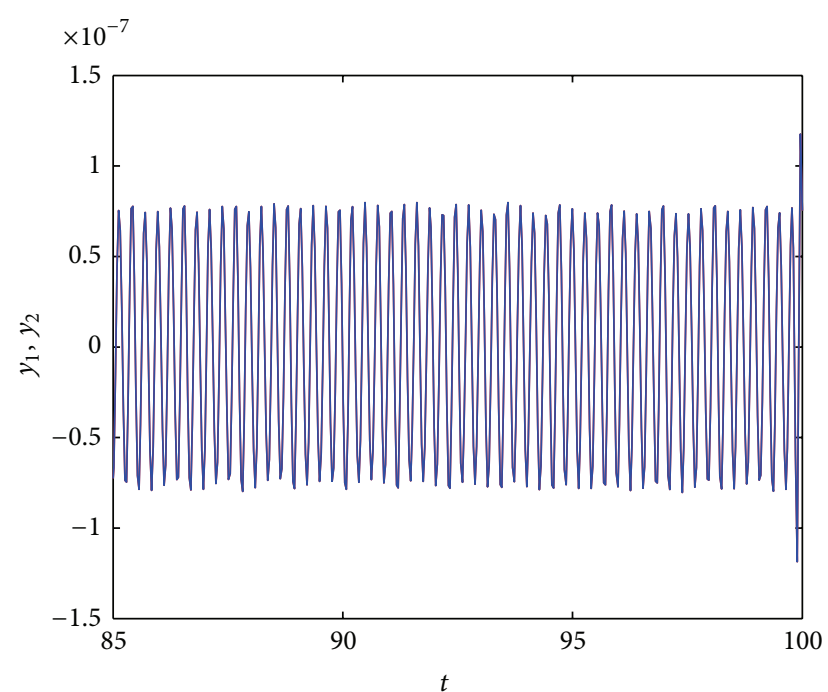

$-y_{1}$

(a)

(b)

FIGURE 1: When $\tau \in\left[0, \tau_{0}^{-}\right]$, the zero equilibrium is unstable. $T=1 / 70$ and initial conditions: $0.000000001 \cdot(1,1,1,1,-1,-1,-1,-1), \tau=0.001$.

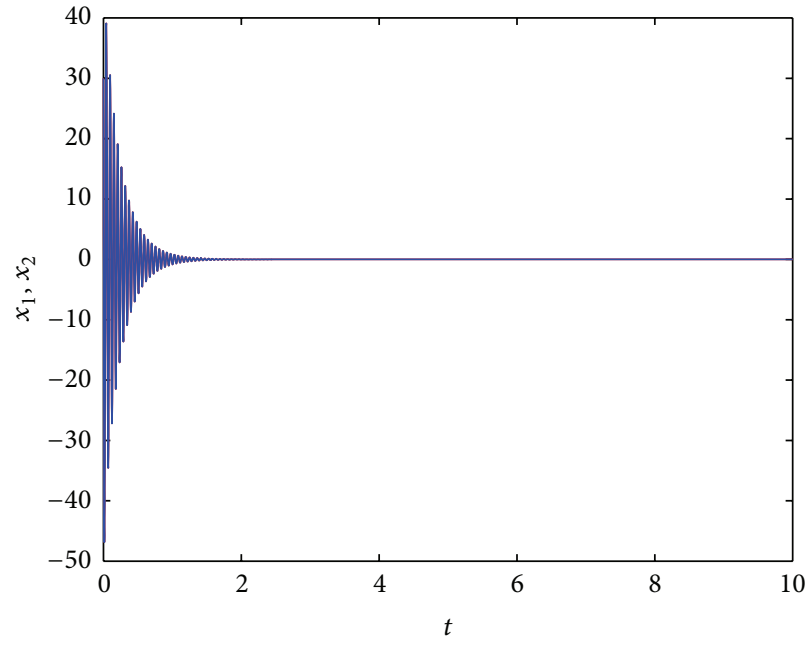

$\begin{aligned} & x_{1} \\ &- x_{2}\end{aligned}$

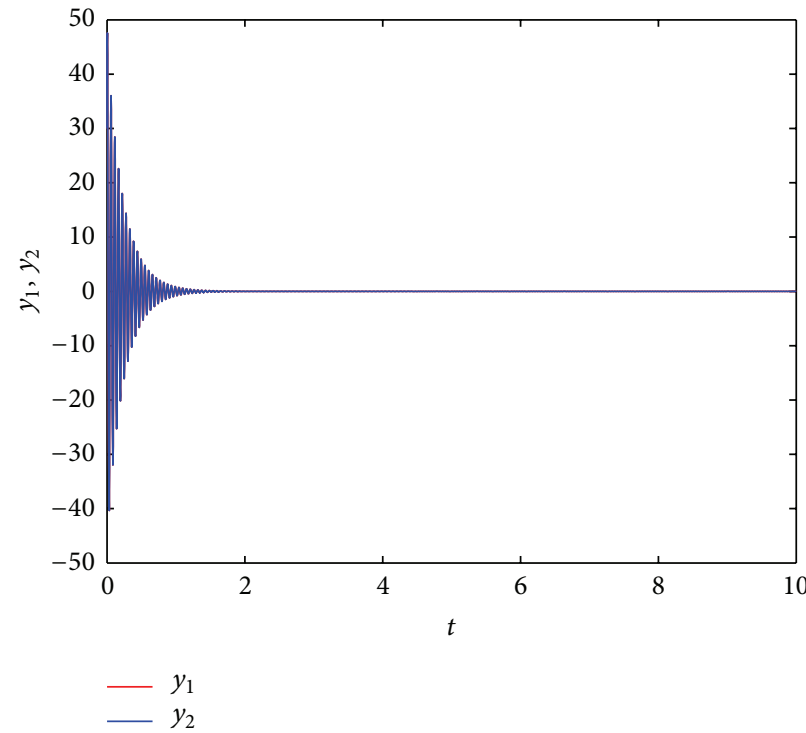

(b)

Figure 2 : When $\tau \in\left[\begin{array}{ll}\tau_{0}^{-} & \tau_{0}^{+}\end{array}\right]$, the zero equilibrium is asymptotically stable. $T=100$ and initial conditions: $30 \cdot(1,1,1,1,1,1,1,1), \tau=0.019$.

Theorem 7. Assume that $\beta_{ \pm}$are defined by (18) and $\tau_{j}^{ \pm}$are defined by (32)-(33), respectively.

(1) If $0<\kappa<P$, then the zero equilibrium of system (3) is unstable for all $\tau \geq 0$.

(2) If $P<\kappa<\sqrt{\alpha^{2} P^{2}+P^{2}}$ and $\arccos (P / \kappa)>$ $\left(\sqrt{\kappa^{2}-P^{2}} /(\alpha P+\Omega)\right) \pi$, then the zero equilibrium of system (3) is stable for $\tau \in \bigcup_{j=0}^{g}\left(\tau_{j}^{-} \tau_{j}^{+}\right)$, and unstable for $\tau \in \bigcup_{j=0}^{g}\left(\tau_{(j-1)}^{+} \quad \tau_{j}^{-}\right) \cup\left(\tau_{g}^{+}+\infty\right)$, where $\tau_{-1}^{+}=0$. In this case, system (3) undergoes a Hopf bifurcation at the zero equilibrium of system (3) when $\tau=\tau_{j}^{ \pm},(j=$ $0,1,2, \ldots)$.

(3) If $P<\kappa<\sqrt{\alpha^{2} P^{2}+P^{2}}$ and $\arccos (P / \kappa)<$ $\left(\sqrt{\kappa^{2}-P^{2}} /(\alpha P+\Omega)\right) \pi$, then the zero equilibrium of system (3) is stable for $\tau \in\left[\begin{array}{ll}0 & \tau_{0}^{+}\end{array}\right) \cup \tau \in$

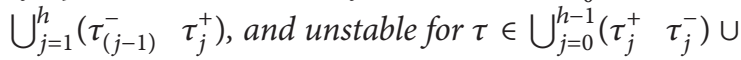
$\left(\tau_{h}^{+}+\infty\right)$. In this case, system (3) undergoes a Hopf bifurcation at the zero equilibrium of system (3) when $\tau=\tau_{j}^{ \pm},(j=0,1,2, \ldots)$. 


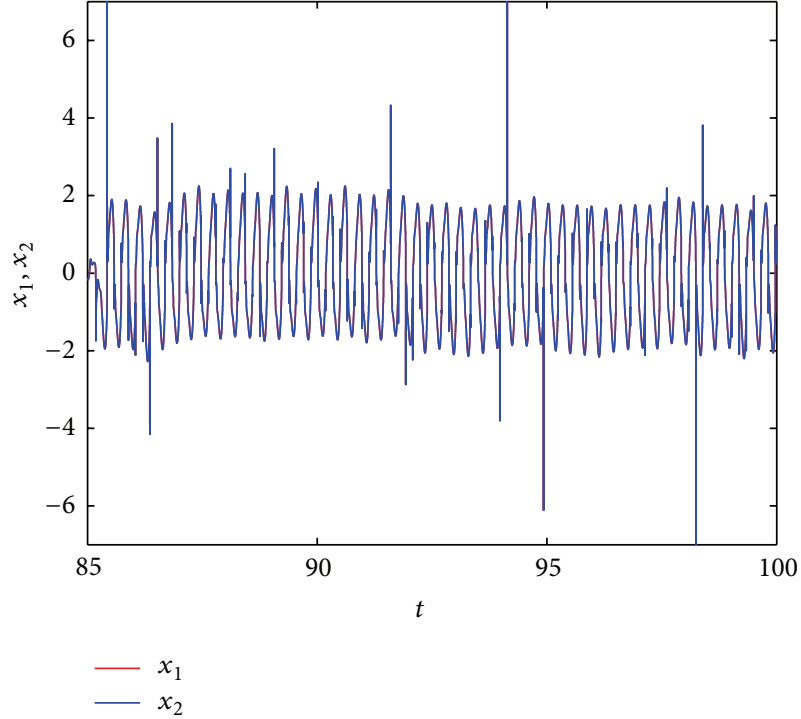

(a)

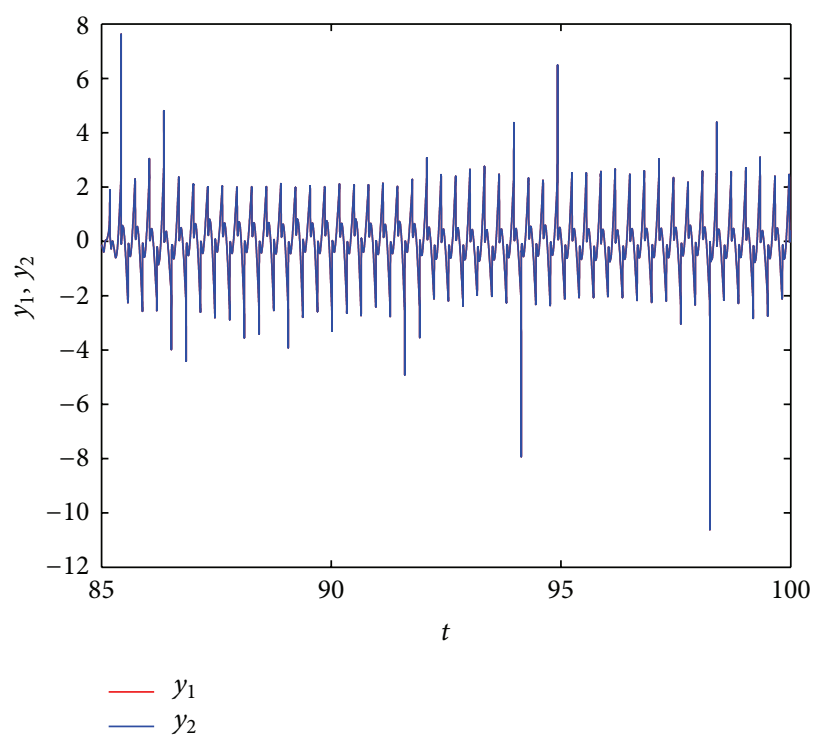

(b)

FIGURE 3: When $\tau \in\left[\begin{array}{ll}\tau_{0}^{+} & \tau_{1}^{-}\end{array}\right]$, the zero equilibrium is unstable. $T=0.01$ and initial conditions: $0.0008 \cdot(1,1,1,1,1,1,1,1), \tau=0.029$.

(4) If $\kappa>\sqrt{\alpha^{2} P^{2}+P^{2}} \bigcap\left(\beta_{-}+\Omega\right)<0$, then the zero equilibriums is unstable for all $\tau \geq 0$, and system (3) undergoes a Hopf bifurcation at the zero equilibrium when $\tau=\bar{\tau}_{j}^{ \pm}$and $\tau=\tilde{\tau}_{j}^{ \pm},(j=0,1,2, \ldots)$. Here $\bar{\tau}_{j}^{ \pm}, \widetilde{\tau}_{j}^{ \pm}$ are shown as Lemmas 1 and 4 , respectively.

\section{Existence of Multiple Periodic Solutions}

In the following, we consider the symmetric properties of (3). Using the theories of functional differential equation, we know that the system (3) is $Z_{2}$-equivariant with

$$
(\rho U)_{r}=U_{r+1}(\bmod 2),
$$

for any $U_{r}$ in $R^{2}$. It is much interesting to consider the spatiotemporal patterns of bifurcating periodic solutions.

In the following, we only consider the periodic properties of $x_{1}(t), y_{1}(t), x_{2}(t), y_{2}(t)$. For this purpose, we give the concepts of some spatiotemporal symmetric periodic solutions. Assume that the state $\left(u_{1}(t), v_{1}(t), u_{2}(t), v_{2}(t)\right)$ can possess two different types of symmetry: spatial and temporal. The oscillators $\left(u_{1}(t), v_{1}(t)\right)$ and $\left(u_{2}(t), v_{2}(t)\right)$ are synchronized if the state taking the form

$$
(u(t), v(t), u(t), v(t)),
$$

for all times $t$. On the other hand, oscillator $\left(u_{1}(t), v_{1}(t)\right)$, is half a period out of phase with (anti-synchronous) oscillator $\left(u_{2}(t), v_{2}(t)\right)$ means the state taking the form

$$
\left(u(t), v(t), u\left(t+\frac{\omega}{2}\right), v\left(t+\frac{\omega}{2}\right)\right) .
$$

Now, we explore the possible (spatial) symmetry of the system (3). Consider the action of $Z_{2} \times S^{1}$ on $\left([-\tau, 0], R^{4}\right)$ with

$$
(r, \theta) x(t)=r x(t+\theta), \quad(r, \theta) \in Z_{2} \times S^{1},
$$

where $S^{1}$ is the temporal. Let $\omega=2 \pi / \beta_{+}$or $\omega=2 \pi / \beta_{-}$, and denote $P_{\omega}$ the Banach space of all continuous $\omega$-periodic function $x(t)$. Denoting $S P_{\omega}$ the subspace of $P_{\omega}$ consisting of all $\omega$-periodic solution of system (3) with $\tau=\bar{\tau}_{j^{ \pm}}^{ \pm}$or $\tau=\widetilde{\tau}_{j^{ \pm}}^{ \pm}$, then for each subgroup $\Sigma \subset Z_{2} \times S^{1}$,

$$
\operatorname{Fix}\left(\Sigma, S P_{\omega}\right)=\left\{x \in S P_{\omega},(r, \theta) x=x, \forall(r, \theta) \in \Sigma\right\} \text {, }
$$

is a subspace.

Theorem 8. Assume that $\beta_{ \pm}$satisfy (18), then near $\tau=\bar{\tau}_{j}^{ \pm}$ ( $\tau=\tilde{\tau}_{j}^{ \pm}$, resp.) for each $j \in N_{0}$, a branch of synchronous (antisynchronous, resp.) periodic solutions of period $\omega$ near $\omega_{0}=$ $\beta_{ \pm} / 2 \pi$ bifurcates from the zero solution of the system (3).

Proof. Let $\beta_{ \pm}$satisfy (18). From the theorem of [11], we know the corresponding eigenvectors of $\Delta^{-}(\lambda)$ at $\tau=\bar{\tau}_{j}^{ \pm}$can be chosen as

$$
q_{1}(\theta)=\left(v_{1}(\theta)^{T}, v_{1}(\theta)^{T}, 1,0,0,0\right)^{T},
$$

where $v_{1}(\theta)$ satisfies $\left[\left(\begin{array}{cc}p & -\alpha p \\ \alpha p & p\end{array}\right)-\left(\begin{array}{cc}\kappa \cos \Omega \bar{\tau}_{j}^{ \pm} & \kappa \sin \Omega \bar{\tau}_{j}^{ \pm} \\ -\kappa \sin \Omega \bar{\tau}_{j}^{ \pm} & \kappa \cos \Omega \bar{\tau}_{j}^{ \pm}\end{array}\right)\right] v_{1}(\theta)=$ $i \beta_{ \pm} v_{1}(\theta)$. The isotropic subgroup of $Z_{2} \times S^{1}$ is $z_{2}(\rho)$, the center space associated to eigenvalues $\pm i \beta_{ \pm}$is spanned by $q_{1}(\theta)$ and $\bar{q}_{1}(\theta)$, and the bifurcated periodic solutions are synchronous, taking the form

$$
(u(t), v(t), u(t), v(t)) .
$$

Similarly, if $\beta_{ \pm}$satisfy (18), then the corresponding eigenvectors of $\Delta^{+}(\lambda)$ at $\tau=\tilde{\tau}_{j}^{ \pm}$can be chosen as

$$
q_{1}(\theta)=\left(v_{1}(\theta)^{T}, v_{1}(\theta)^{T}, 1,0,0,0\right)^{T},
$$




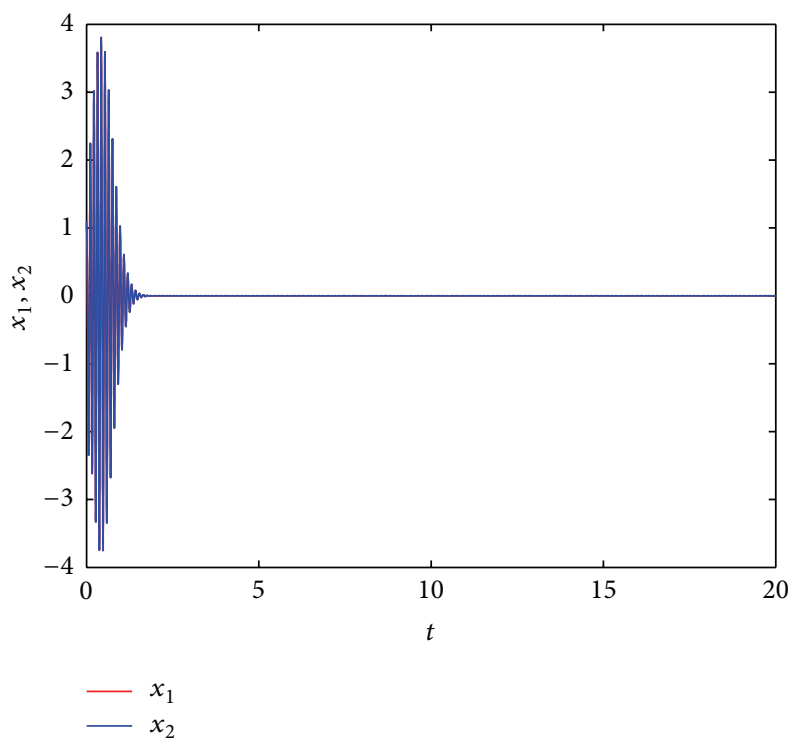

(a)

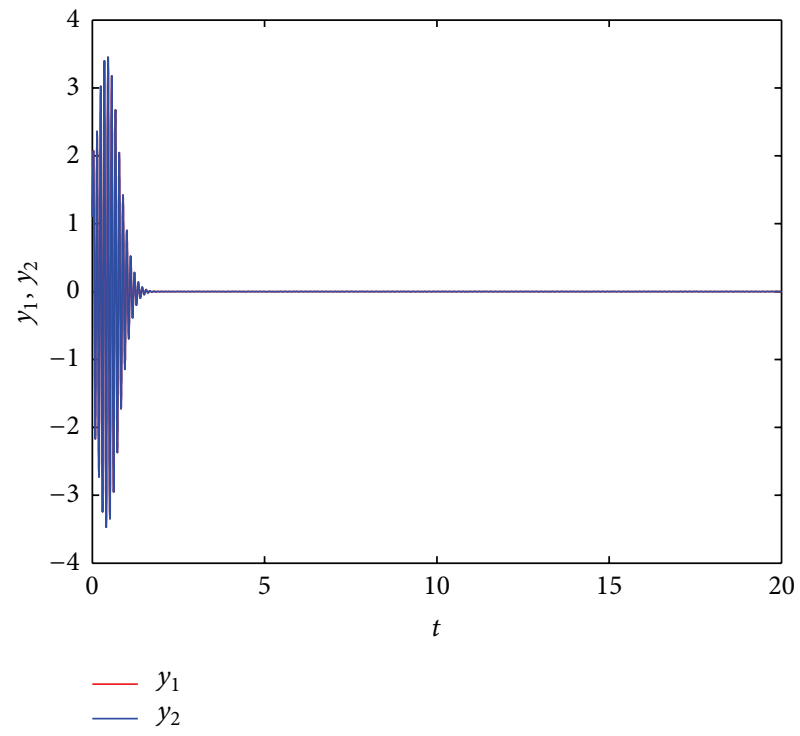

(b)

FIGURE 4 : when $\tau \in\left[\begin{array}{ll}\tau_{1}^{-} & \tau_{1}^{+}\end{array}\right]$, the zero equilibrium is asymptotically stable. $T=1$ and initial conditions: $1.1 \cdot(1,1,1,1,1,1,1,1), \tau=0.0299$.

and $v_{2}(\theta)$ satisfies $\left[\left(\begin{array}{cc}p & -\alpha p \\ \alpha p & p\end{array}\right)+\left(\begin{array}{cc}\kappa \cos \Omega \widetilde{\tau}_{j}^{ \pm} & \kappa \sin \Omega \tilde{\tau}_{j}^{ \pm} \\ -\kappa \sin \Omega \widetilde{\tau}_{j}^{ \pm} & \kappa \cos \Omega \tilde{\tau}_{j}^{ \pm}\end{array}\right)\right] v_{1}(\theta)=$ $i \beta_{ \pm} v_{2}(\theta)$.

$Z_{2} \times S^{1}$ has another isotropic subgroup $z_{2}(\rho, \pi)$, the center space associated to eigenvalues $\pm i \beta_{ \pm}$is spanned by $q_{2}(\theta)$, $\bar{q}_{2}(\theta)$, and the bifurcated periodic solutions are anti-phased, that is, taking the form

$$
\left(u(t), v(t), u\left(t+\frac{\omega}{2}\right), v\left(t+\frac{\omega}{2}\right)\right),
$$

where $\omega$ is a period.

\section{Numerical Simulations}

As we all know that when $\Omega_{1} \neq \Omega_{2}$, model (1) has been studied by Erzgraber et al. According to their results, when changing the detuning between the lasers they observed that the coupled laser system undergoes mode jumps to other stable compound laser mode within the locking region.

In this paper, we consider the properties of stability and bifurcation in a mutually delay-coupled semiconductor lasers system. By analyzing the associate characteristic equation, we can determine the stability and bifurcation of the coupled system (3) with a single delay in four cases of Theorem 7. Specifically, we have shown case (2) in Theorem 7 by using some numerical simulations; then the Hopf bifurcation occurs as $\tau$.

Let $\kappa=25, P=20, \Omega=275$, and $\alpha=2$, through (32) and (33) we have

$$
\begin{aligned}
\tau_{0}^{-} & =\frac{\pi-\arccos (4 / 5)}{\beta_{-}+\Omega}=0.0188 \\
& <\tau_{0}^{+}=\frac{\pi+\arccos (4 / 5)}{\beta_{+}+\Omega}=0.0210
\end{aligned}
$$

$$
\begin{aligned}
& <\tau_{1}^{-}=\frac{2 \pi-\arccos (4 / 5)}{\beta_{-}+\Omega}=0.0293 \\
& <\tau_{1}^{+}=\frac{2 \pi+\arccos \left(\frac{4}{5}\right)}{\beta_{+}+\Omega}=0.0305, \ldots .
\end{aligned}
$$

Theorem 7 shows that the zero equilibrium of system (3) is unstable for $\tau \in\left[\begin{array}{ll}0 & \tau_{0}^{-}\end{array}\right] \cup\left[\begin{array}{ll}\tau_{0}^{+} & \tau_{1}^{-}\end{array}\right] \cup\left[\begin{array}{ll}\tau_{1}^{+} & \infty\end{array}\right]$ and asymptotically stable for $\tau \in\left[\tau_{0}^{-}, \tau_{0}^{+}\right] \cup\left[\tau_{1}^{-}, \tau_{1}^{+}\right]$. This means that as the average delay $\tau$ varies, the zero equilibrium of system (3) switches two times from instability to stability, then to instability as shown in Figures 1,2,3, and 4, and finally becomes unstable.

\section{Conclusion}

In our study, we give a semiconductor lasers model with coupled delay to describe the exchanges between the two optical fields $E_{1}, E_{2}$ of the lasers. By means of the general symmetric local Hopf bifurcation theorem, we not only investigated the effect of delay of signal transmission on the pattern formation of model (3) but also obtained some important results about the spontaneous bifurcation of multiple branches of periodic solutions and their spatiotemporal patterns. From a practical viewpoint, these means that the time delay could cause a stable equilibrium to become unstable and cause the properties in a coupled semiconductor lasers system to fluctuate: if $\tau<\tau_{0}$, the output power of the lasers reach equilibrium. If $\tau$ increases and crosses the value $\tau_{0}$, then this equilibrium becomes unstable and the output power has a change of periodic and small amplitude. 


\section{Acknowledgment}

This research was supported by the National Natural Science Foundations of China.

\section{References}

[1] A. P. S. Dias and J. S. W. Lamb, "Local bifurcation in symmetric coupled cell networks: linear theory," Physica D, vol. 223, no. 1, pp. 93-108, 2006.

[2] D. Fan and J. Wei, "Hopf bifurcation analysis in a tri-neuron network with time delay," Nonlinear Analysis: Real World Applications, vol. 9, no. 1, pp. 9-25, 2008.

[3] S. Guo and L. Huang, "Hopf bifurcating periodic orbits in a ring of neurons with delays," Physica D, vol. 183, no. 1-2, pp. 19-44, 2003.

[4] C. Zhang, B. Zheng, and L. Wang, "Multiple Hopf bifurcations of three coupled van der Pol oscillators with delay," Applied Mathematics and Computation, vol. 217, no. 17, pp. 7155-7166, 2011.

[5] K. Miyakawa and K. Yamada, "Entrainment in coupled saltwater oscillators," Physica D, vol. 127, no. 3-4, pp. 177-186, 1999.

[6] H. Erzgräber, D. Lenstra, B. Krauskopf et al., "Mutually delaycoupled semiconductor lasers: mode bifurcation scenarios," Optics Communications, vol. 255, no. 4-6, pp. 286-296, 2005.

[7] L. Illing, G. Hoth, L. Shareshian, and C. May, "Scaling behavior of oscillations arising in delay-coupled optoelectronic oscillators," Physical Review E, vol. 83, no. 2, Article ID 026107, 2011.

[8] F. Drubi, S. Ibáñez, and J. A. Rodríguez, "Coupling leads to chaos," Journal of Differential Equations, vol. 239, no. 2, pp. 371385, 2007.

[9] J. Wu, "Symmetric functional-differential equations and neural networks with memory," Transactions of the American Mathematical Society, vol. 350, no. 12, pp. 4799-4838, 1998.

[10] M. Golubitsky, I. Stewart, and D. G. Schaeffer, Singularities and Groups in Bifurcation Theory. Vol. II, vol. 69 of Applied Mathematical Sciences, Springer, New York, NY, USA, 1988.

[11] C. Zhang, Y. Zhang, and B. Zheng, "A model in a coupled system of simple neural oscillators with delays," Journal of Computational and Applied Mathematics, vol. 229, no. 1, pp. 264-273, 2009. 


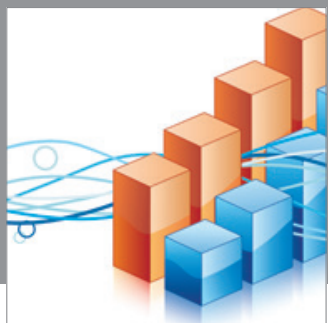

Advances in

Operations Research

mansans

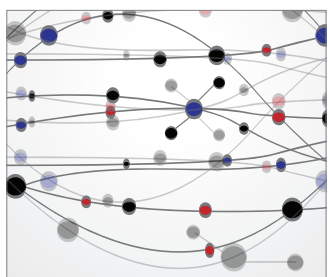

The Scientific World Journal
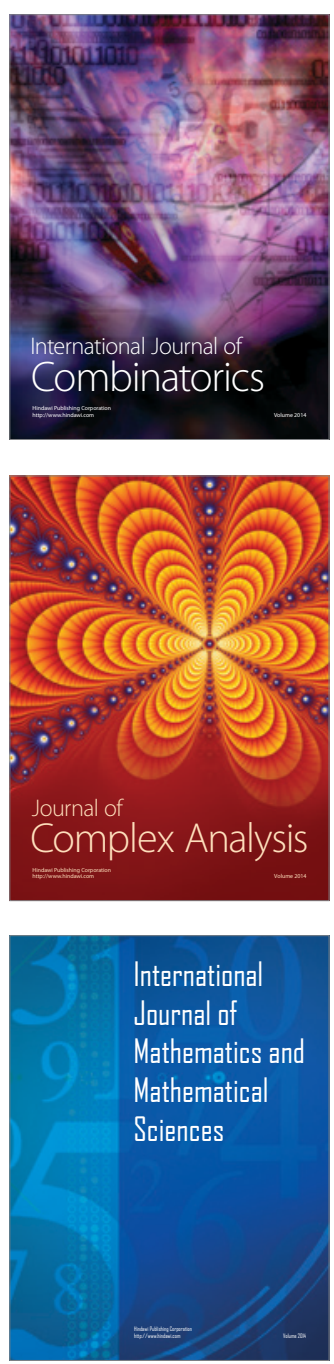
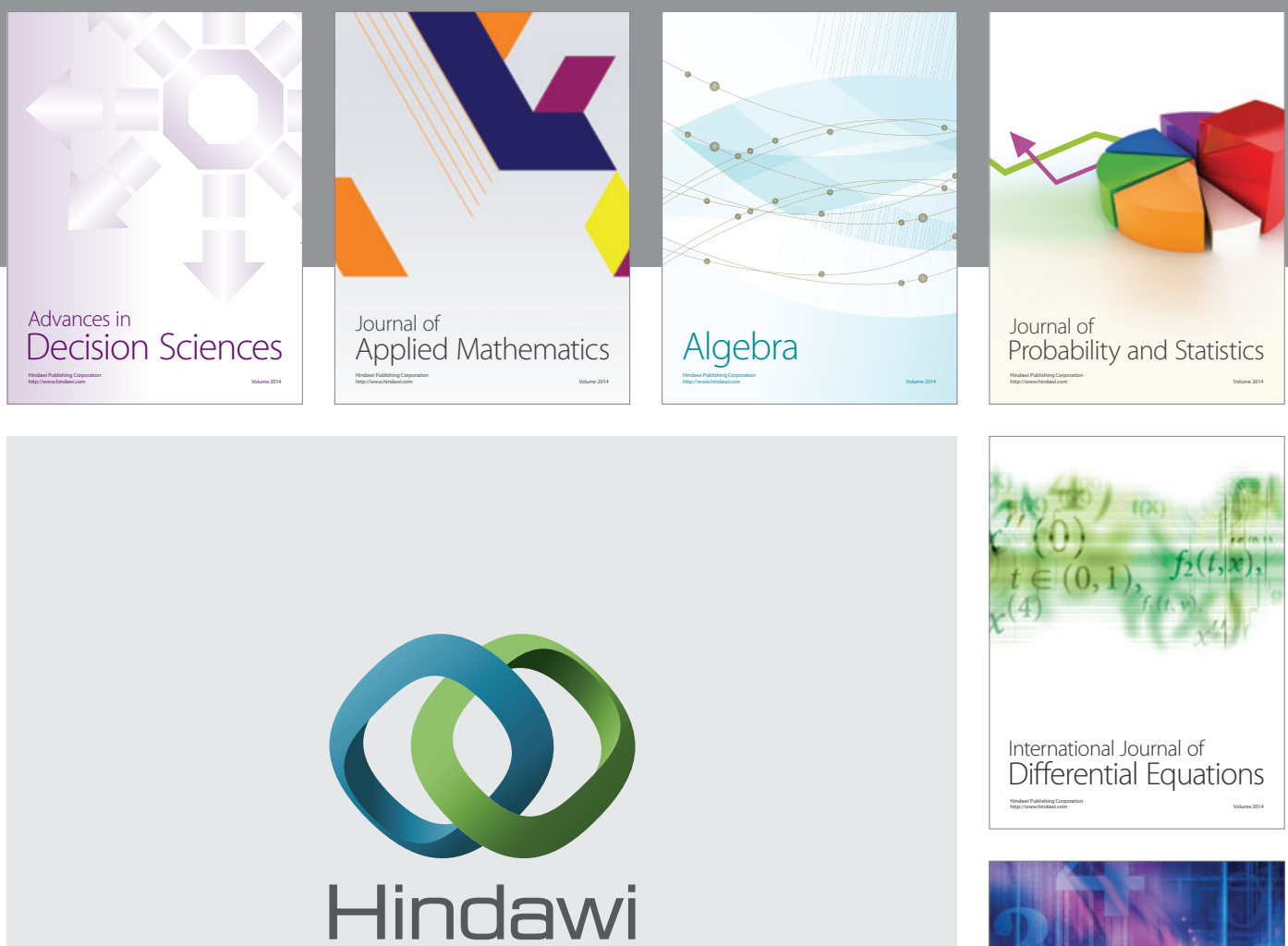

Submit your manuscripts at http://www.hindawi.com
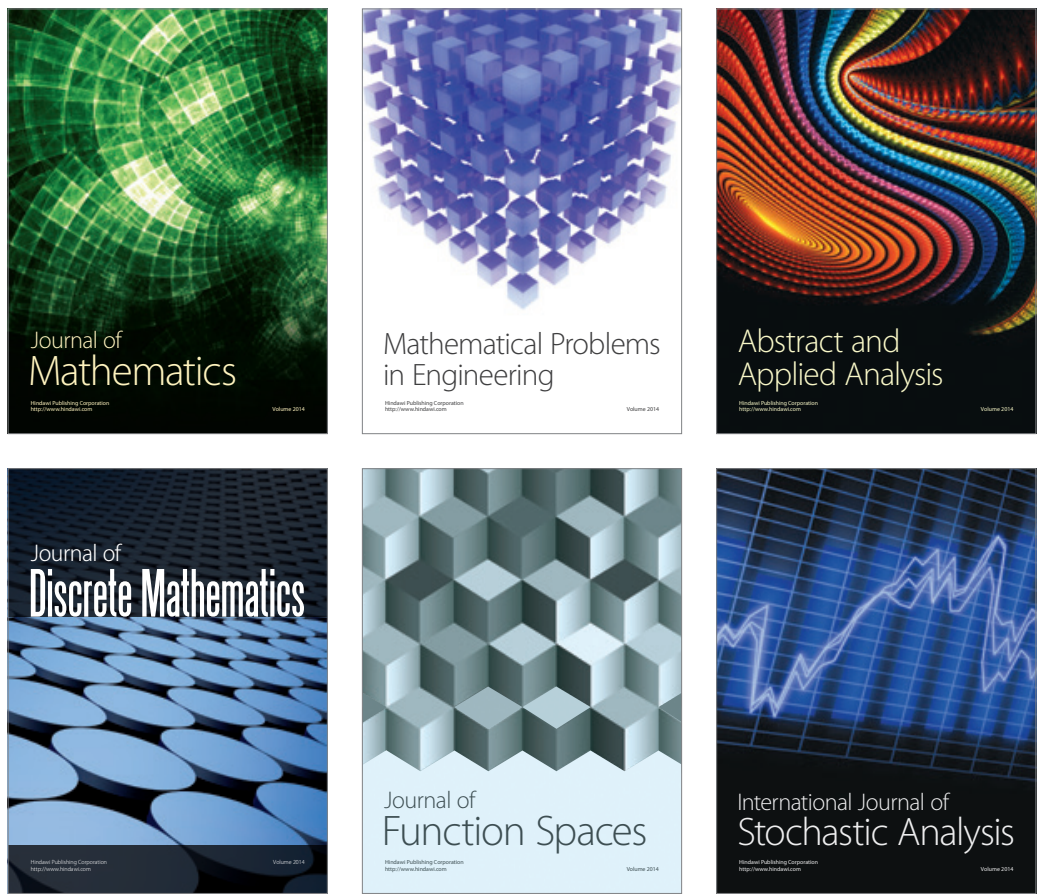

Journal of

Function Spaces

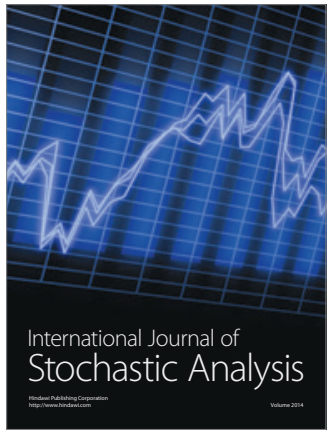

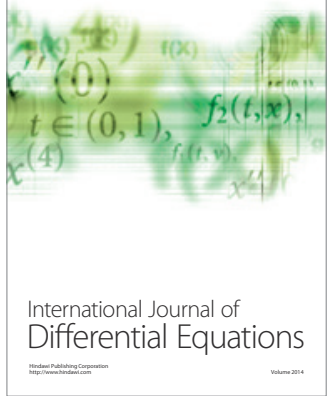
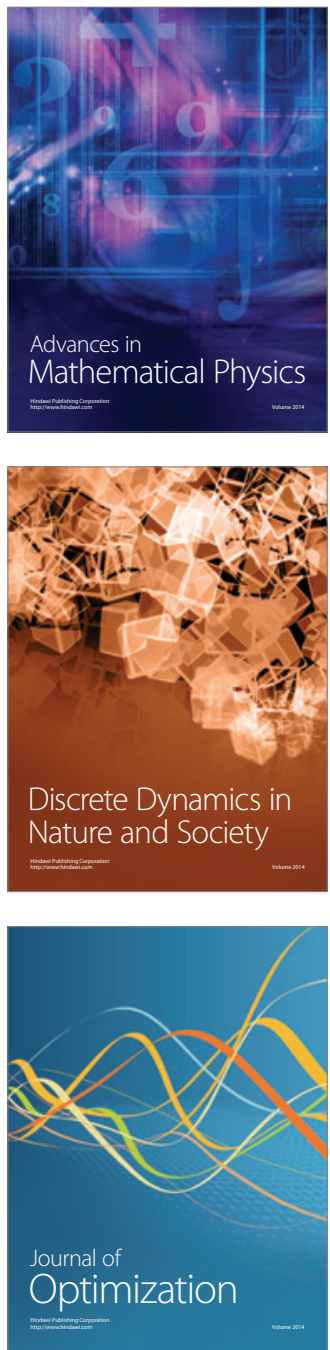